\title{
Lithium in metal-poor red giants
}

\author{
Laimons Začs and Arturs Barzdis \\ Faculty of Physics and Mathematics, University of Latvia, \\ Raiņa bulvāris 19, LV-1586 Rīga, Latvia \\ email: zacs@latnet.lv
}

\begin{abstract}
The lithium abundance was calculated for five metal-poor red giant stars from Li I doublet at $6707 \AA$ by fitting the observed high-resolution spectra with synthetic spectra. The lithium abundance was found to be low in all stars, $\log \varepsilon(\mathrm{Li}) \leqslant 1.8$, confirming lithium depletion on the red giant and asymptotic giant branch.
\end{abstract}

Keywords. Stars: abundances, Population II, late-type, binaries: spectroscopic, stars: individual (HD 30443, HD 187216, HD 209621, HD 218732, HD 232078)

\section{Observations and analysis}

High resolution spectra for three analyzed stars (HD 209621, HD 218732, HD 232078) were observed with the cross-dispersed high-resolution échelle spectrograph FIES installed on NOT (Canary Islands) with a spectral resolution of $\mathrm{R}=67000$. HD 187216 was observed using the fiber-fed échelle spectrograph BOES attached to the 1.8-m telescope of Bohyunsan Observatory (Korea) with a spectral resolution of $\mathrm{R}=45000$. HD 30443 was observed using the coudé échelle spectrometer MAESTRO on the $2 \mathrm{~m}$ telescope at the Observatory on the Terskol Peak in Northern Caucasus (Russia) with a resolving power of 45000 . The basic data for all targets are provided in Table 1. Spectral classification was adopted from Yamashita (1975) and the SIMBAD database. All stars are first-ascent red giants or on the asymptotic giant branch. HD 30443 and HD 209621 are spectroscopic long period binaries $(\mathrm{P}=2954 \& 407.4$ days; McClure \& Woodsworth 1990). HD 218732 was suspected to be a long period binary ( $\mathrm{P}=567$ days; Carney et al. 2003).

Synthetic spectra for some lithium abundances around the final value are calculated and convolved with the instrumental profile using spectrum synthesis technique which based on the R.L.Kurucz's code SYNTHE and the Uppsala atmospheric models. Figure 1 illustrates iterations $( \pm 0.1$ dex for HD 30443, \pm 0.2 dex for HD 187216, HD 209621, and HD 232078, \pm 0.5 dex for HD 218732) around the accepted lithium value. The accepted lithium abundances are provided in Table 1. The line list was examined using the databases VALD and DREAM. Significant blending of Li doublet was expected because of low effective temperature and peculiar chemical composition of stellar atmospheres. $C_{2}$ and CN lines are strong in the spectra of HD 30443, HD 187216, and HD 209621 (see Figure 1) and lines of neutron capture elements are enhanced.

\section{Conclusions}

Five red giants in the metallicity range $-1<[\mathrm{Fe} / \mathrm{H}]<-2$ display $\log \varepsilon(\mathrm{Li}) \leqslant 1.8$, confirming a substantial lithium depletion on the red giant and asymptotic giant branch. Neutron capture elements are significantly enhanced in the amospheres of three analyzed giants. 
Table 1. Basic data for the program stars. Accepted effective temperature (K), gravity (cgs), iron abundance relative to the solar value, lithium abundance and averaged abundance of neutron capture elements relative to the solar value are given.

\begin{tabular}{cccccccc}
\hline Star & Sp.type & $T_{\text {eff }}$ & $\log g$ & {$[\mathbf{F e}$ I/H $],[\mathbf{F e}$ II $/ \mathbf{H}]$} & $\log \varepsilon(\mathbf{L i})$ & {$[\mathbf{n} / \mathbf{F e}$ II $]$} & binary \\
\hline HD30443 & R, C4,3 CH & 4100 & 1.5 & $-1.1,-1.1$ & 1.8 & +1.6 & yes \\
HD187216 & R, C3,3 CH & 4000 & 0.75 & $-2.6,-1.7$ & 0.45 & +1.2 & \\
HD209621 & C, C1,2 CH & 4300 & -0.4 & $-2.0,-2.0$ & 1.2 & +1.3 & yes \\
HD218732 & G7 Ib & 4200 & 0.50 & $-1.5,-1.5$ & -1.1 & 0.0 & yes \\
HD232078 & K3 IIp & 4000 & 0.00 & $-1.4,-1.4$ & -0.55 & +0.3 & \\
\hline
\end{tabular}

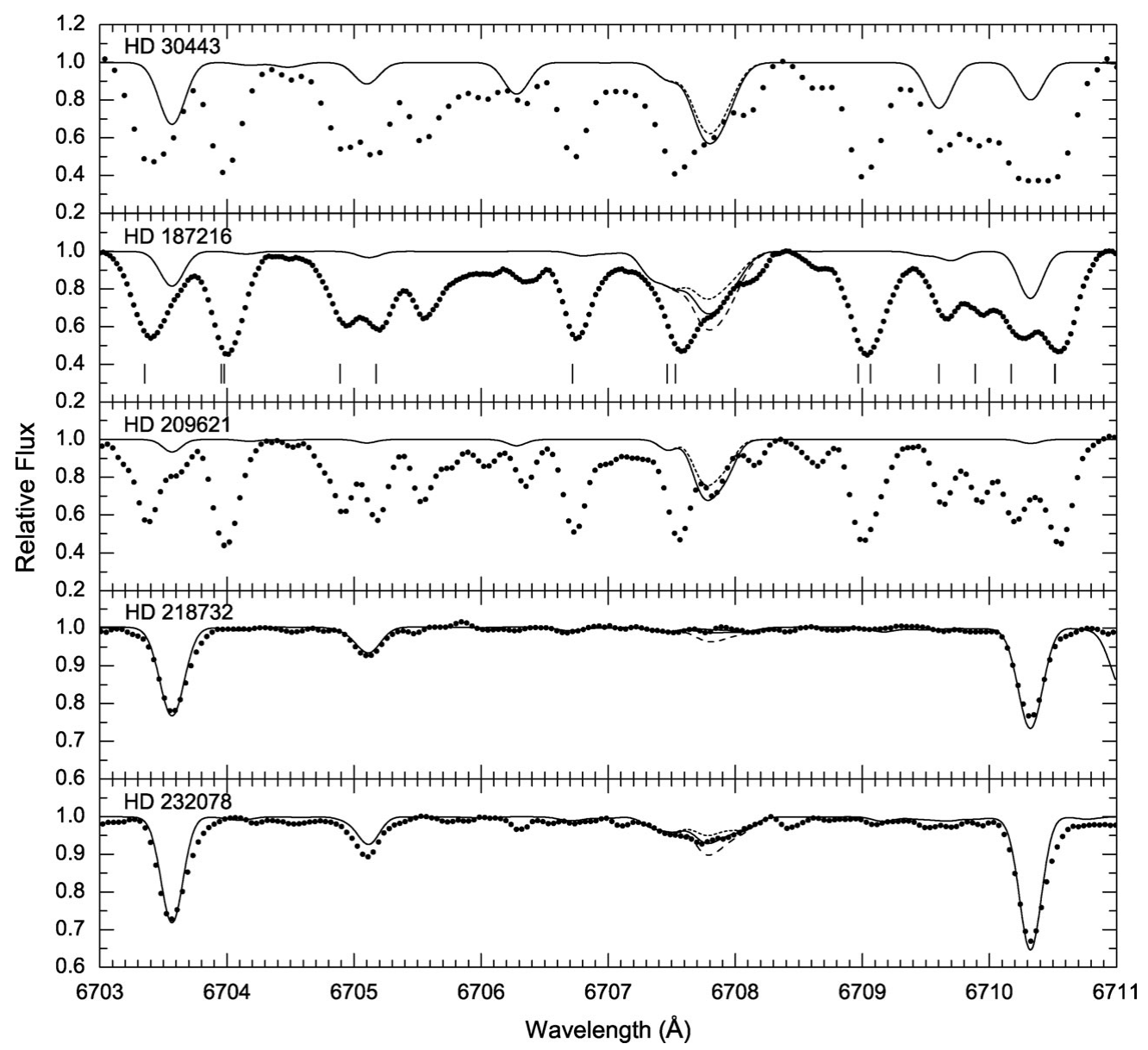

Figure 1. The observed spectra (dots) of red giants in spectral region around Li doublet at $6707 \AA$. The positions of CN Red system lines are marked for HD 187216. Synthesized atomic line spectra for the accepted lithium abundances are displayed by thick solid lines.

\section{References}

Carney, B. W., Latham, D. W., Stefanik, R. P., Laird, J. B., \& Morse, J. A. 2003, AJ, 125, 293 McClure, R. D. \& Woodsworth, A. W. 1990, ApJ, 352, 709

Yamashita, Y. 1975, PASJ, 27, 325 